JSCMT

Journal of Sustainable Construction

Materials and Technologies

J. Sustain. Construct. Mater. Technol. 2(2) (2017) 132-137
Journal of Sustainable

Construction Materials and Technologies

http://www.eds.yildiz.edu.tr/jscmt

\title{
Evaluation of Urban Transport with International Examples
}

\author{
Zeynel Doğru ${ }^{1, \mathrm{a}, *}$, Mehmet Fatih Altan ${ }^{1, \mathrm{~b}}$ \\ ${ }^{l}$ Faculty of Engineering, Istanbul Aydin University, Florya Campus, Istanbul, Turkey \\ azeyneldogru@stu.aydin.edu.tr, ${ }^{b}$ mehmetaltan@aydin.edu.tr
}

Received November 6, 2016; accepted January 21, 2017

\begin{abstract}
The story of urbanization and transportation is as ancient as human history. Throughout history, people have been established their habitats near rivers, intersection points of roads and trade routes in alignment with strategical, cultural points or martial policies. Almost all of these are directly or indirect transportation elements. One needs to evaluate samples from different geographies comparatively to realize planning improvement. In this context, Turkey's cities, especially major of them such as Istanbul, Ankara, İzmir, and Bursa do not manifest uniform topographies and characteristics. Hence, it is beneficial to evaluate cities in other locations which resemble Turkey's cities to obtain different perspectives.
\end{abstract}

Keywords: Economy; Mass Transportation; Infrastructure; Transportation System; Urbanization

\section{Introduction}

Transportation and urbanization are the driving force behind the interrelated and continuous relationship between technical, economic, social, cultural, political and military disciplines.

Therefore, they affect the future development of economics, politics, socio-culture, and security of rural areas and surroundings, cities, districts, many other regions, and even whole countries directly and positively or negatively.

Since the establishment of the Republic, Turkey has been experiencing rapid urbanization. However, a correct urbanization process has never occurred due to infrastructure problems, insufficient planning, lack of balanced distribution, integration, and coordination.

Migration from rural to urban areas, from Anatolia to the western region continued at an accelerating rate in every period. On a global scale, these issues still prevail. In this sense, the analyzing European cities alone will not be sufficient. Thus, this paper delves into the dynamics in developing countries such as China and India, which make up a significant portion of the world in terms of land and population.

\footnotetext{
* Corresponding author.

E-mail address: zeyneldogru@stu.aydin.edu.tr

https://doi.org/10.29187/jscmt.2018.17
} 


\section{The Scales of China and India}

Cities in China and India suffer from severe and worsening transportation problems, such as traffic blockage, parking shortages, noise, air pollution, high energy usage and lack of mobility mostly for low-income earners and traffic accidents resulting in major injuries and deaths,

Major problems in the crisis of urban transport in both China and India are intractable population growth, the growth of disorganized and chaotic urbanization, urbanization itself, the rapid increase in car ownership and usage due to steadily increasing income.

On one hand, a steady increase in the use of motor vehicles is an expected condition. On the other hand, tax increases, payments, and pricing of motor vehicles that reflect great environmental and social costs should restrict the use of motor vehicles in large urban centers with traffic congestion.

At the same time, more stringent regulations must be applied to produce cleaner, quieter, safer and more energy efficient vehicles for public and personal transportation.

When combined, China's and India's populations correspond to $37 \%$ of the world's population, ranking both countries as the first and most important developing countries in the world. Despite the rather low level of per capita income in these countries, a considerable increase in income has been achieved thanks to the rapid economic development in the past decade.

Between 1980-2005, per capital real income (adapted to inflation) in India has more than doubled, and in China has increased by more than four times. High-income growth drives the rapid increase in ownership and usage of motor vehicles in both countries.

Since 1990, the total number of motor vehicles in India has tripled, while the total number of motor vehicles has increased by ten times in China, causing growing alarming levels in traffic accident-induced injuries and deaths, air pollution, traffic congestion, noise and high energy usage [1].

\section{Similarity and Differences between China and India}

While China and India are similar in many ways, they differ in several important points. Urban transportation systems can be taken as one of the basic criteria for the differences and similarities of both the countries.

Many of these similarities between China and India are present in other developing countries, where some of these factors indicate common characteristics.

The differences between China and India indicates the changes in the economic, political and transport systems of developing countries in a concrete manner. In this context, progressive steps of urbanization, land usage, travel behavior, transportation problems, and the recent developments in government policies and the factors in question have been addressed in detail. Both the similarities and the differences in China and India can help to elucidate the structure of transportation problems and general policies of developing countries.

Both China and India have witnessed significant population growth in the recent past, but the speed of population growth in India is higher. In fact, from 1989 to 2005, India's annual population growth rate is almost twice that of China's annual population growth rate during the same period (India 1,7 \%, China 0,9\%).

In proportion to India, the comparatively low population growth of China is derived from solid family population planning policies that allow a family to have at most one child. These policies are only suspended in some few exceptional cases.

In both countries, especially in China, population growth is concentrated in urban areas, which is caused by domestic migration due to economic pressures in rural areas. While the urban population in China increased from 178 million in 1978 to 524 million in 2003 (about 4.4\% increase), the urban population in India increased from 160 million in 1981 to 285 million in 2001. In this case, while the total population growth is faster in India, China is still ahead in urban population growth. 
For both countries, up-to-date urban population growth may exceed the official statistics because a significant number of persons not registered in census counts are temporarily dwelling in cities (often low-income population migrated from rural areas) [2].

In both China and India, big cities are the focal point of population and development. Both countries have three cities each with a population of 10 million or even more: In China; Beijing (12.4 million), Shanghai (15.4 million), Chongqing (15.2 million) and in India; Mumbai (16.4 million), Calcutta (13.2) and Delhi (12.8 million). In addition, there are five cities (Guangzhou, Tianjin, Xi'an, Chengduve, Wuhan) with a population of 5 to 10 million in China and three cities (Chennai, Hyderabad, Bangalore) with a population of 5 to 10 million in India.

Counting cities with a population of 1 million and over, the number of such cities in China is 174, while in India the number is 35 [2].

The rapid development of cities in both China and India has significantly increased the demand for land and travel in urban areas, which has brought the pressure on transportation and other infrastructure types to very high levels. The continuous increase in urban population alone is enough to cause serious transportation problems.

However, with the large increase in average income, especially in China motorization rates immediately started to climb. While the per capita national income showed an 8-fold increase in China between 1972-2002, the corresponding value showed only a two-fold increase in India for the same years.

In 1972, the per capita national income in China was $43 \%$ of that in India, while in 2002, the per capita national income in China reached a $46 \%$ higher rate than the per capita national income of India in 2002. Income growth triggered an increase in the ownership and use of private vehicles and motorcycles in both countries [3].

In parallel with the population growth, we see the expansion of Chinese and Indian cities into suburban areas of relatively lower population density. For example, while the developed area of Chinese cities increased more than three times from 1985 to 2003 (from $9386 \mathrm{~km} 2$ to $28.308 \mathrm{~km} 2$ ), the total urban population doubled.

\section{Land Use}

The Chinese suburbs are generally less populated than the more historical parts of the country, but more populated than North American suburbs. In China, public transport is better planned and coordinated with higher levels of service so that people are less dependent on cars. Due to the lack of affordable housing in the central areas of Chinese cities and a lack of a clear government policy for rejuvenation of urban areas, in China, most low- and middle-income households tend to leave for the surrounding suburbs.

During the urban development process, many factories are also moved to suburban areas. Thus employment is evenly distributed between the city centers and the suburbs. In addition, local Chinese governments are encouraging the establishment of new industrial and technological parks in the surrounding areas, which is driving urban development towards rural areas of the country [4].

A similar decentralization process of cities and surrounding areas is also seen in India, which is partly due to conscious government policies aimed at the reduction of crowding in urban centers.

Land use regulations severely limit the proportion of residential areas to the total area in urban centers, which is related to the height and the increase in density of the buildings in those urban centers.

With the growth of Indian cities, new developmental effects are emerging towards the suburban borders. Also, the land use regulations laid down by local administrative laws in the suburbs are more stringent than those in cities, even encouraging and promoting policies that allow the economic development of urban centers to be distributed.

Similar to China, almost all major Indian cities have large technology parks at their outer city borders, which encourages both population and employment to be distributed from the city center to the surrounding areas. 
While suburban developments around Chinese cities are often planned and coordinated to some degree with the provision of basic public infrastructures, Indian cities are often unplanned, lacking provision of adequate basic public infrastructure and sufficient public transport services.

In China and India cities, decentralization affects urban transportation to a large extent. The growth of cities in both countries increases the length of travel in most urban areas, leading to a more comprehensive travel demand, which results in more traffic on public transport systems and roads. Increasing travel distances also make walking and cycling a less convenient way to travel encouraging the transition from non-motorized to motorized transport vehicles.

\section{Urban Transportation Problems and Trends}

The share of public transportation generally rises parallel to the increasing population volume. Using discrete categories of urban size, the Indian Ministry of Transport recorded an average public transport usage rate of 63\% in cities with a population of 5 million more and only $16 \%$ in cities with populations of 100,000-250,000.But there are no noteworthy differences in the urban size categories [5].

While Delhi's mode of public transportation is almost the same as that of medium-sized cities such as Chennai, Hyderabad, and Bangalore, it has scant bus services as in smaller towns of Kanpur and Luck now.

China's two largest cities, Beijing and Shanghai both have a $25 \%$ mode share of public transportation. Population development, suburban expansion and the construction of subway systems in these cities indicate that the use of public transport and industrial share will increase in these cities in the near future.

From 1999 to 2004, for example, the mode of share of public transport in Shanghai rose from 15\% to 24\% according to the Chinese Ministry of Construction.

The most surprising public transport share is in Tianjin with 8\%.The low public transport rate in Tianjin, which is the center of China's bicycle industry, is probably due to its extraordinary high rate of bicycle usage (51\% of all trips).

In both, China and India, there is an alarming rise in traffic-related deathsover the last 30 years. Despite the fact that population growth is controlled, traffic-related death rates have increased five times in China and three times in India. Since 2003, there were 105,000 recorded fatal traffic accidents in China, compared to 80,000 in India. This corresponds to 128.3 traffic deaths per 100,000 motor vehicles in China, 108.1 for India.

These numbers are five times higher than those in the United States (USA). In the USA, the number of fatal accidents per 100,000 motor vehicles was recorded as only 18.5 during the same year.[5]

\section{Traffic Congestion}

Affecting all modes of transport and all socio-economic groups, traffic congestion on a daily basis is perhaps the most visible, the most common and the most urgent transportation problem in the cities of both China and India.

Many calculations, as well as anecdotal indicators, predict rapidly worsening traffic congestion. In Mumbai, for example, from 1962-1993 the average road speed of motor vehicles decreased from $38 \mathrm{~km} / \mathrm{h}$ to $15-20 \mathrm{~km} / \mathrm{h}$. In Delhi, the average motor vehicle speed dropped from 20-27 km / h in 1997 to $15 \mathrm{~km} / \mathrm{h}$ in 2002 . Also, peak congestion periods in Delhi are from 08:30 to 10:30 in the morning and from 16:30 to 19:30 in the afternoon, lasting a total of about 5 hours. The average speed in Cennay is $13 \mathrm{~km} / \mathrm{h}$, whereas the average speed in Calcutta varies from 10 to $15 \mathrm{~km} / \mathrm{h}$, but falls to $7 \mathrm{~km} / \mathrm{h}$ in the city center. Highway speeds also continue to decrease in Chinese cities. In Beijing, the average motor vehicle speed decreased from $45 \mathrm{~km} / \mathrm{h}$ in 1994 to only $12 \mathrm{~km} / \mathrm{h}$ in 2003 , where the effect of traffic congestion is also seen in bus speeds, with the average operating speed down from $17 \mathrm{~km}$ / h in 1997 to $9 \mathrm{~km} / \mathrm{h}$ in 2003 [6]. 
Traffic congestion has a time-consuming and disabling effect on passengers. Both Chinese and Indian cities are growing outward, and the average travel distance is increasing.

Along with lower travel speeds, the expansion of the suburbs increases the average travel time by significant amounts. Average total times to and from work in suburban settlements of large cities in India have now increased up to 3 hours/day.

Chinese cities have not yet experienced the enlargement of the suburbs at the level of Indian cities, but the average expansion durations of the suburbs are in a definite and constant rise.

\section{Results}

To sum up, the subject of preexisting heavy transport problems in the cities of China and India, which are intensifying day by day, has been discussed. Problems of transportation are chiefly due to rapidly increasing urbanization, increase in motorization and partly to low income.

Near-term policies in China and India usually focus on promoting best-motorized transport to wealthy populations to stimulate economic growth by providing all motorized transport modes. As one of the most effective means of ensuring the sustainability of economic development, the incentivation of the motor vehicle manufacturing industry illustrates the official policy of both countries.

Central and regional governments favor tax reduction, subsidies and concession arrangements for the increase in industrial profitability.

In addition, governments at all levels focus on increasing road network capacity to meet the increased demand for motorized transport modes, especially the demand for cars and trucks. Figure 1 below shows the population density of world cities [7].

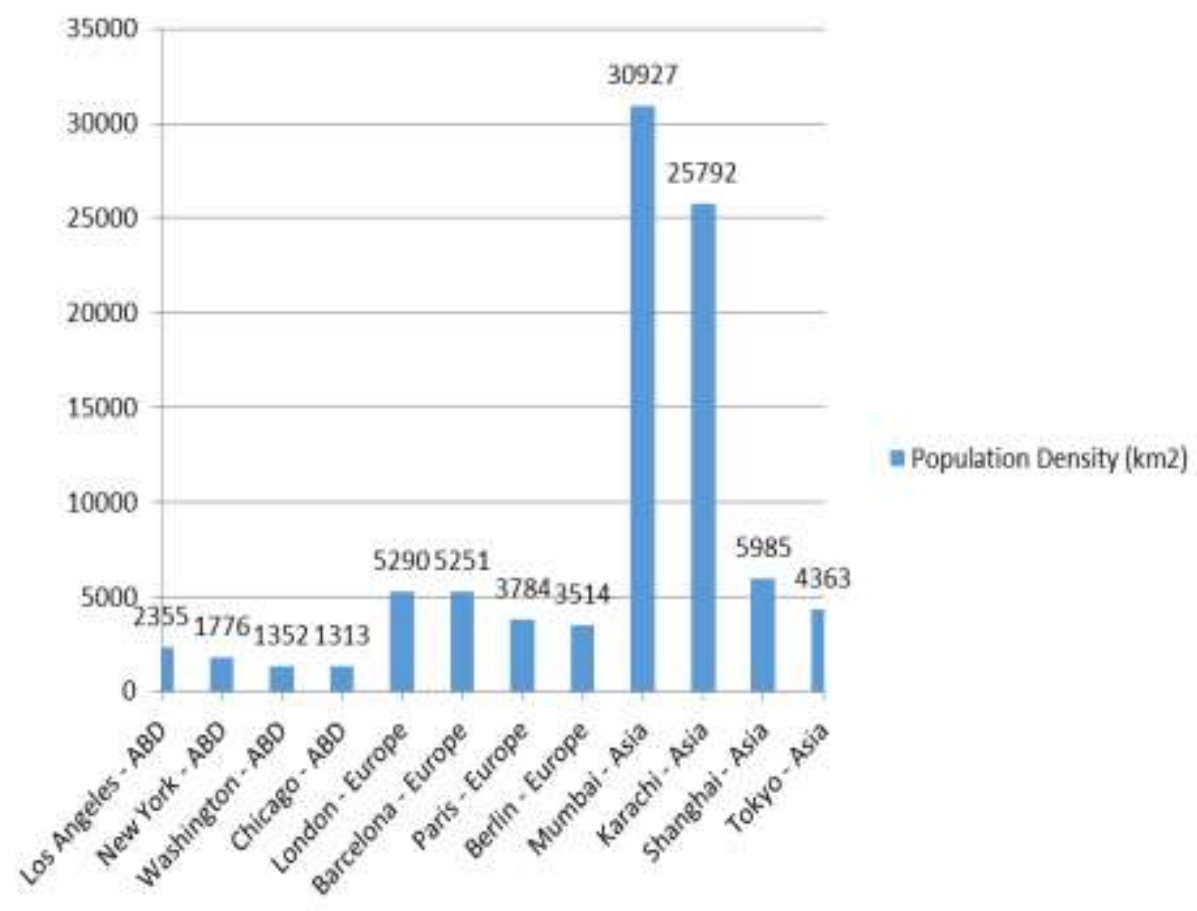

Fig 1. Population Density in World Cities [7] 
Both Chinese and Indian cities are rapidly decentralizing towards the surrounding areas. Up to a point, this is a natural consequence of population growth and the need to utilize suburban areas for new settlement and commercial development. In Indian cities, suburbanization trends are accelerating, intensified by less stringent construction standards in suburban areas and by limited urban population densities. At the same time, Chinese cities are also moving away from the center, but this is less salient than in India due to stricter government control over the structure of settlement and suburban development in China.

\section{References}

1. Acharya, R. (2000). Indian Railways: where the commuter is king? Japan Railways and Transport Review, 25, pp. 34-45.

2. Badami, M., Tiwari, G. and Mohan, D. (2004). Access and mobility for the urban poor in India: bridging the gap between policy and needs. Paper presented at the Forum on Urban Affairs, Delhi, India.

3. Beijing City Planning Institute (2001). Report of 2000 Household Trip Survey in Beijing (Beijing: Beijing City Planning Institute).

4. Beijing Transportation Commission (2004). The Olympic Economy and Infrastructure Construction Stimulate the Development of the Market in Beijing. Available at: http://www.bjitw.gov.cn/ file.jsp?wzbh=743\&lmbh=7

5. Bhat, S. (2003) Noise and the law, India Together, November. Available at: http://www.indiatogether.org/2003/nov/law-noise.htm

6. He, K., Huo, H., Zhang, Q., He, D., An, F., Wang, M. and Walsh, M. (2005). Oil consumption and CO2 emissions in China's road transport: current status, future trends, and policy implications, Energy Policy, 33, pp. 1499-1507.

7. Agarwal, O. P. (2001). Towards a national urban transport policy. Indian Journal of Transport Management 25 (6): 593-616. 